\title{
Design of diblock co-oligomers as low bandgap small molecules for organic solar cells
}

\section{Eliezer Fernando Oliveira \& Francisco Carlos Lavarda}

To cite this article: Eliezer Fernando Oliveira \& Francisco Carlos Lavarda (2017) Design of diblock co-oligomers as low bandgap small molecules for organic solar cells, Molecular Simulation, 43:18, 1496-1501, DOI: 10.1080/08927022.2017.1321759

To link to this article: https://doi.org/10.1080/08927022.2017.1321759

View supplementary material ๔

Published online: 02 May 2017.

Submit your article to this journal $\pi$

Џll Article views: 82

View Crossmark data $\asymp$

4 Citing articles: 2 View citing articles 준 


\title{
Design of diblock co-oligomers as low bandgap small molecules for organic solar cells
}

\author{
Eliezer Fernando Oliveira ${ }^{a}$ and Francisco Carlos Lavarda ${ }^{a, b}$ \\ aPOSMAT - Graduate Program in Material Science and Technology, UNESP - Univ Estadual Paulista, Bauru, Brazil; bDF-FC, UNESP - Univ Estadual Paulista, \\ Bauru, Brazil
}

\begin{abstract}
The properties of a particular kind of small molecule that is built from two oligomers of different monomers, i.e. a diblock co-oligomer, as the electron donor in the active layer of organic solar cells are investigated theoretically. For these molecules, this work shows that it is possible to predict the energies of the frontier molecular orbitals by knowing the same energies for the oligomers that constitute the diblock, opening the possibility of designing new materials with optimal energy levels and optical properties. Furthermore, it was observed that the optical absorption bands of these diblock co-oligomers were broader than that of the constituent oligomers and also of the homopolymers, allowing greater absorption of photons and possibly an improved electric current in the device. It was also shown that these phenomena are sizedependent.
\end{abstract}

ARTICLE HISTORY

Received 28 February 2017

Accepted 18 April 2017

\section{KEYWORDS}

Computational modelling; DFT; diblock co-oligomers; optical properties; organic solar cells

\section{Introduction}

New materials for organic solar cells (OSCs) must have properties such as good solubility, stability and other features that allow a good morphology of the active layer and high production at low cost [1-3]. In addition, the materials used in the active layer of such devices should also have properties that increase the efficiency of solar energy conversion [4]. In particular, the new electron donor material should provide: (i) an adjustment of the energies of the lowest unoccupied and highest occupied molecular orbitals (LUMO and HOMO, respectively) in relation to the acceptor materials, in order to ensure a higher open circuit voltage $\left(V_{\mathrm{OC}}\right)$; (ii) a higher electric current via a wider photon collection, resulting from a small energy difference between HOMO and LUMO $\left(\Delta E_{\mathrm{HL}}\right)$; and (iii) an ability to promote the exciton dissociation, avoiding recombination [1-5].

Although most of the active layers of bulk heterojunction OSCs are made from polymers and fullerenes, small molecules have been employed as electron donor materials due to several advantages such as easy synthesis and high purity $[3,5,6]$. Efficiencies around $10 \%$ were reported for solar cells with small molecules as electron donors and fullerenes derivatives as electron acceptor materials [6,7]. The drawback commonly found in these materials is lower values for the fill factor $(\mathrm{FF})$ that are related to morphology problems [6,7]. The use of several new small molecules has been proposed in the literature, usually built from electron donor and electron acceptor constituent units (DA concept) $[3,8-12]$. This approach has proven to be effective to obtain new small molecules with HOMO and LUMO energies ( $E_{\mathrm{HOMO}}$ and $E_{\mathrm{LUMO}}$, respectively) more adjusted to the adopted electron acceptor material, thereby providing an optimised $V_{\mathrm{OC}}$. However, the $\Delta E_{\mathrm{HL}}$ still have values above those suggested as ideal (around $1.4 \mathrm{eV}$ ) $[3,13]$.

In this paper a proposal is presented to design small organic molecules as electron donor materials. Making use of theoretical chemistry calculations, it will be shown that for a small molecule that is built from two oligomers of different monomers, i.e. a diblock co-oligomer (DCO), it is possible to predict the energies of the frontier molecular orbitals by knowing beforehand the same energies for the oligomers. Based on a long series of results, we concluded that the DCO's $E_{\text {HOMO }}$ and $E_{\mathrm{LUMO}}$ can be estimated as approximately equal to the $E_{\text {Номо }}$ of the oligomer that has the highest energy for the HOMO orbital and the $E_{\text {LUMO }}$ of the oligomer that has the lowest energy for the LUMO orbital, respectively (see Figure 1). This concept allows that from a collection of oligomers with known $E_{\mathrm{HOMO}}$ and $E_{\mathrm{LUMO}}$, it is possible to plan a new electron donor molecule with the best possible fit of the frontier electronic levels both in relation to the acceptor material to be used as well as to the lowest $\Delta E_{\mathrm{HL}}$. And the results show that there is still the bonus of a broader optical absorption band than the simple overlap of the optical absorption bands of the constituent oligomers. Such a scheme opens the possibility of saving efforts to find new materials that bring together good electronic and optical properties that can be predicted, and also good morphology and charge transport, which can normally only be evaluated after the device is built.

In the following sections, in order to show the plausibility of our proposal, we present our study of a DCO consisting of oligomers of thiophene and pyrrole, $[\mathrm{Th}]_{\mathrm{n}}$-block- $[\mathrm{Py}]_{\mathrm{n}}$, since 


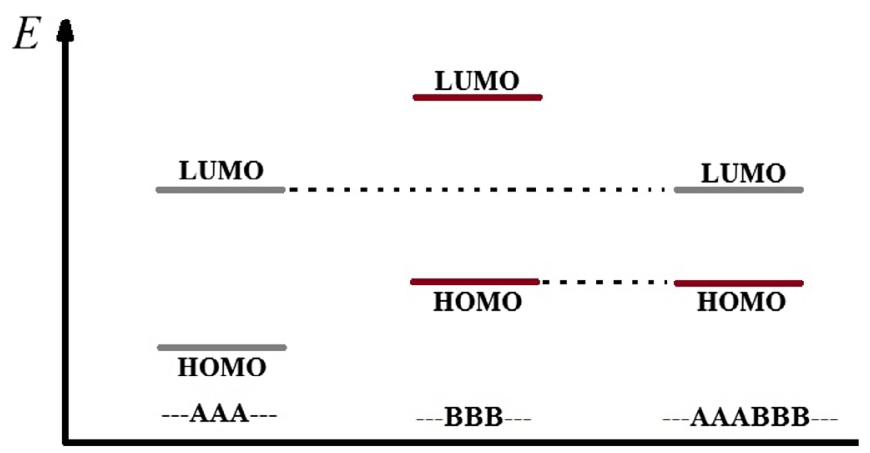

Figure 1. (Colour online) Frontier electronic energy levels for oligomers $[A]_{n^{\prime}}$ $[B]_{m}$ and for the diblock co-oligomer built from $[A]_{n}$ and $[B]_{m^{\prime}}$ according to the predicting rule for them.

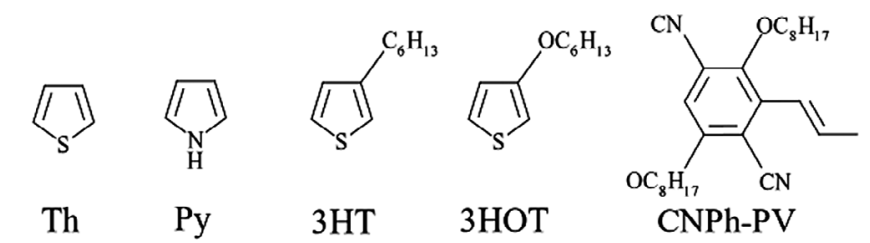

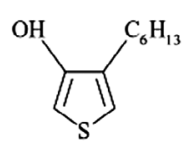

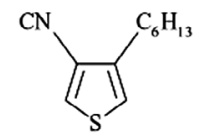

3HT-OH
$3 \mathrm{HT}-\mathrm{CN}$

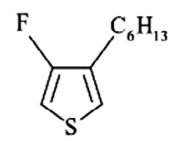

3HT-F the other monomers, for which we already knew the frontier electronic energy levels from previous works [16-18]. Based on the electronic properties of the isolated oligomers, the chosen combination for the DCOs are: $\mathrm{Th}+\mathrm{Py}, \mathrm{CNPh}-\mathrm{PV}+3 \mathrm{HOT}$, $3 \mathrm{HT}-\mathrm{OH}+3 \mathrm{HT}-\mathrm{CN}, 3 \mathrm{HT}-\mathrm{F}+3 \mathrm{HT}-\mathrm{CN}, 3 \mathrm{HT}-\mathrm{NH}_{2}+3 \mathrm{HT}-\mathrm{CN}$ e $3 \mathrm{HT}+3 \mathrm{HT}-\mathrm{CN}$.

We decided to use DCOs bonding together two different oligomers with the same number of monomeric units. Thus, if we have two monomers, $A$ and $B$, our DCOs are of the type $[A]_{n}-$ block- $[\mathrm{B}]_{\mathrm{n}}$. The number $\mathrm{n}$ of monomeric units was varied from 2 to 10 in order to analyse the electronic properties' dependence on the size of the DCO. We adopted oligomeric chains with 20 monomers to represent the polymer, because usually for this oligomer size the electronic properties are very close to the polymer limit [20-23].

All geometry optimisations were carried out in vacuo using the Hatree-Fock method PM6 [24] with the MOPAC2012 package [25]. Next, we obtained the electronic structure data using the Density Functional Theory (DFT) [26], employing the B3LYP hybrid functional $[27,28]$ and the basis functions set $6-31 \mathrm{G}(\mathrm{d})$ [29] with the GAUSSIAN09 program [30]. There are today a large amount of available functionals and theoretical studies have shown that B3LYP provides in average a very good agreement with the experimental values for optical and electronic properties in studies with organic small molecules and polymers [31-36], whereas coulomb attenuated and long-range separated functionals generally overestimate these values $[32,35]$. To reduce computational costs, we replaced the long side chains of the studied structures (octoxy of CNPh-PV, hexyloxy of 3HOT, and hexyl of $3 \mathrm{HT}$ and its derivatives) with methyl groups, an approach that has been shown to be feasible, as the electronic and optical properties basically remain unchanged [37]. Such an approach of combining a geometry optimised at a semiempirical level of theory and the electronic properties through DFT calculations has been applied with satisfactory results in conjugated polymer studies $[17,18,37-39]$. This methodology makes our study possible, as exclusive use of ab initio methods would have resulted in a high computational cost because of the size of the systems and mainly the number of conformers analysed.

We adopted fully planar oligomeric structures with anticonformation, since this is the most probable conformation pointed out in theoretical and experimental studies $[17,20,21,39,40]$ and is the expected conformation in the solid state $[20,41,42]$. As the polymers and oligomers selected for this study already tend to be planar, it was assumed that the same conformation holds for the DCOs, an option also supported by experimental works on small molecules [5,32]. We also did some tests optimising the structures without constraints, i.e. not restricted to a planar conformation; the results obtained showed that the structures became slightly twisted with variations smaller than $0.1 \mathrm{eV}$ for the energy of the frontier electronic levels between the twisted and those totally planar structures. These results suggest that employing full planar structures is a realistic approach.

In addition to the electronic structure of oligomers and DCOs, we also evaluated the allowed optical transitions between the ground and excited states of the studied materials. The optical absorption calculations, represented by the energies of the allowed vertical transitions $\left(E_{\text {vert }}\right)$, were performed through the Time Dependent Density Functional Theory (TD-DFT) [43] the main idea of this work. This is the same reason to choose 
with the same functional, basis set and software described above. Orbital pictures were drawn with Jmol [44].

\section{Results and discussions}

For the reasons presented in the preceding Section, our most detailed study is of the Th and Py oligomers and the DCOs built from them. As the same conclusions were observed in all cases, the main results for the other DCOs are presented in the Supplemental Online Material (SOM).

The $[\mathrm{Th}]_{\mathrm{n}}$ and $[\mathrm{Py}]_{\mathrm{n}}$ homo-oligomers ranged from 2 to 10 monomeric units, and from these we built DCOs. According to Figure 3, the homo-oligomers present $E_{\mathrm{HOMO}}$ and $E_{\mathrm{LUMO}}$ values reasonably different and one intense optical absorption peak relating to the HOMO $\rightarrow$ LUMO transition (see Figure S1 of SOM). It is noted that the $[\mathrm{Th}]_{\mathrm{n}}$-block- $[\mathrm{Py}]_{\mathrm{n}}$ DCOs have $E_{\mathrm{HOMO}}$ and $E_{\mathrm{LUMO}}$ values approximately equal to the $E_{\mathrm{HOMO}}$ of the $[\mathrm{Py}]_{\mathrm{n}}$ and to the $E_{\mathrm{LUMO}}$ of the $[\mathrm{Th}]_{\mathrm{n}}$ oligomers, respectively. The same effect is observed for the other studied DCOs, as can be seen in Figures S3, S6, S9, S12, and S15 of SOM. This set of results corroborates the rule of prediction of the frontier electronic levels of DCOs presented in the Introduction. As a consequence, the $\Delta E_{\mathrm{HL}}$ can be modelled, choosing oligomers with the appropriate $E_{\text {HOMO }}$ and $E_{\text {LUMO }}$ values. In the case of Th and Py monomers, the values of $\Delta E_{\mathrm{HL}}$ of the DCOs are always lower than the constituents oligomers.

To present the results of the theoretical optical absorption of the DCOs of Th and Py, the case $[\mathrm{Th}]_{5}$-block- $[\mathrm{Py}]_{5}$ will be taken as an example; the other studied cases can be seen in Figure S1 of SOM. Figure 4 shows the optical absorption spectrum of the DCO, together with the spectra of homo-oligomers [Th $]_{5}$ and $[\mathrm{Py}]_{5}$. It is possible to observe that the DCO's first optical transition has a lower energy compared to the homo-oligomers, as the calculated $\Delta E_{\mathrm{HL}}$ already indicated. We also note that the spectrum of $[\mathrm{Th}]_{5}$-block- $[\mathrm{Py}]_{5}$ is broader and shows about four

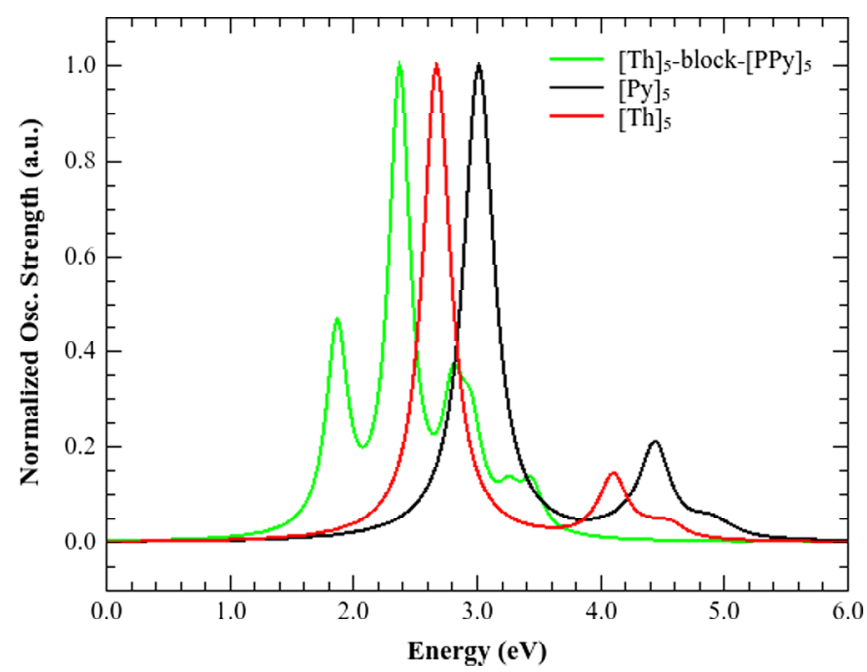

Figure 4. (Colour online) Theoretical optical absorption spectra of 5 monomeric units of Py (red) and Th (blue) compared to [Th $]_{5}$-block-[Py $]_{5}$ (black).

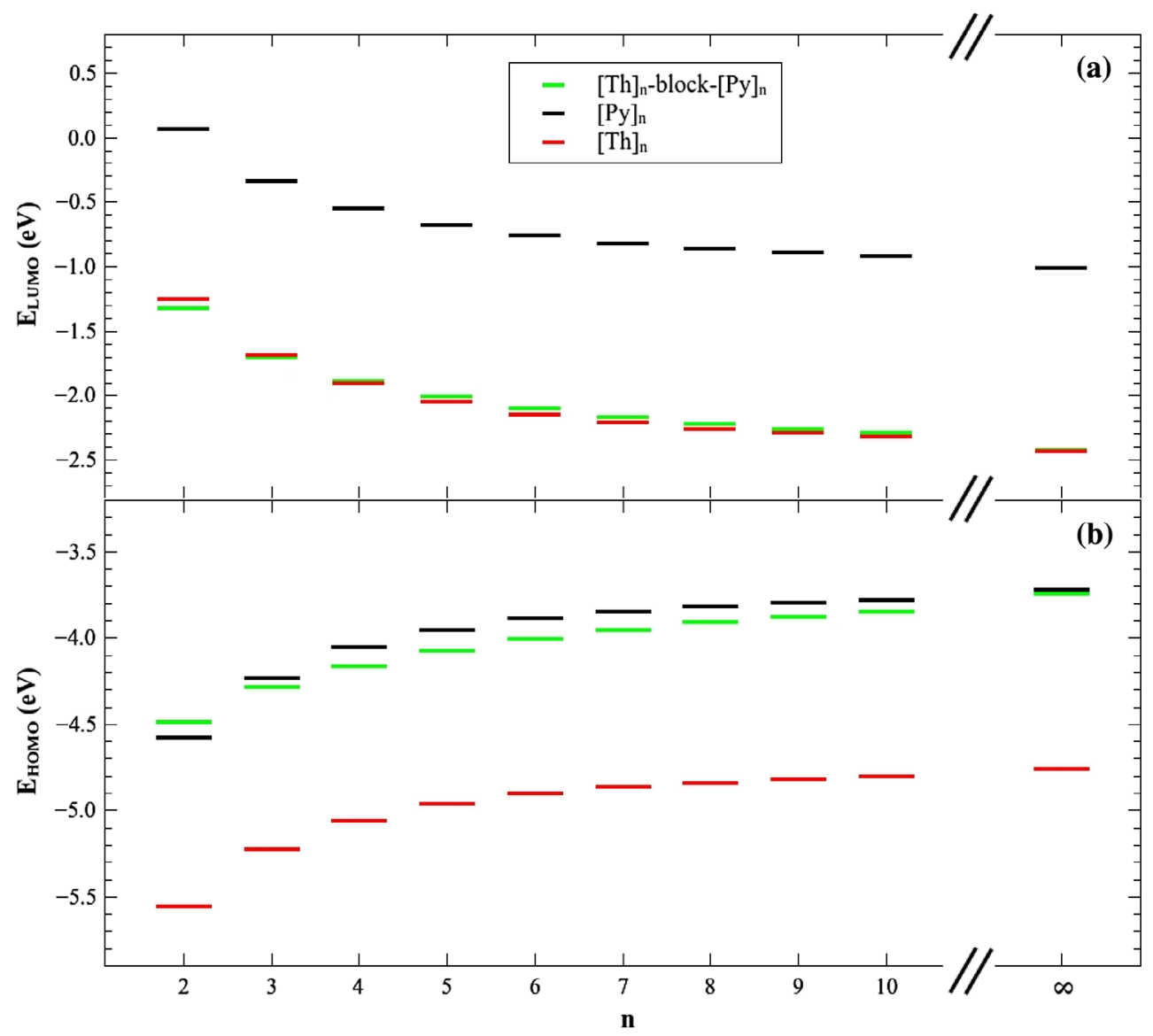

Figure 3. (Colour online) (a) $E_{\text {LUMO }}$ and (b) $E_{\text {номо }}$ for $[T h]_{n}$ (red), $[\mathrm{Py}]_{n}$ (black) and $[\mathrm{Th}]_{n}$-block-[Py $]_{n}$ (green). 
$[\mathrm{Th}]_{5}$-block-[Py $]_{5}$
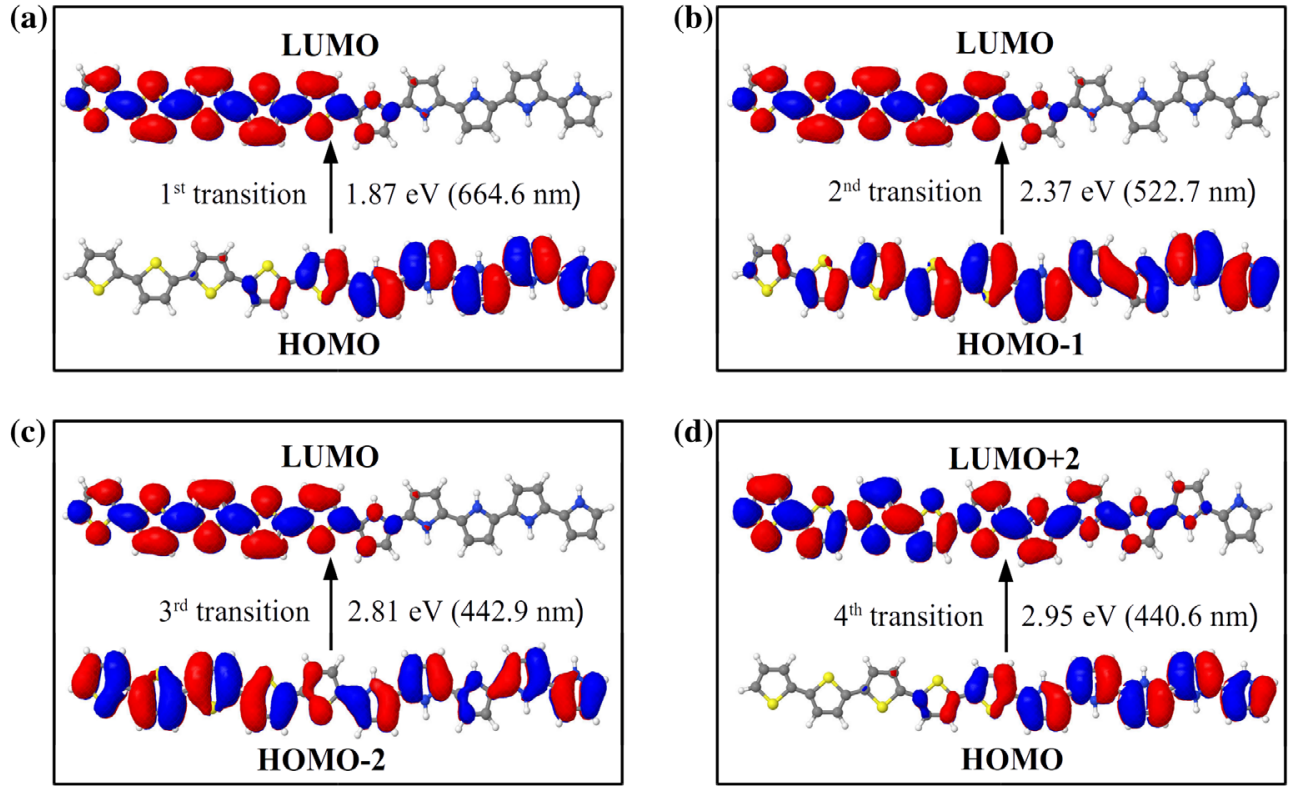

Figure 5. (Colour online) Orbitals (Kohn-Sham representation, isovalue surface of $0.01 \mathrm{au}$ ) involved on the (a) first, (b) second, (c) third and (d) fourth most intense transitions for $[\mathrm{Th}]_{5}$-block-[Py $]_{5}$.

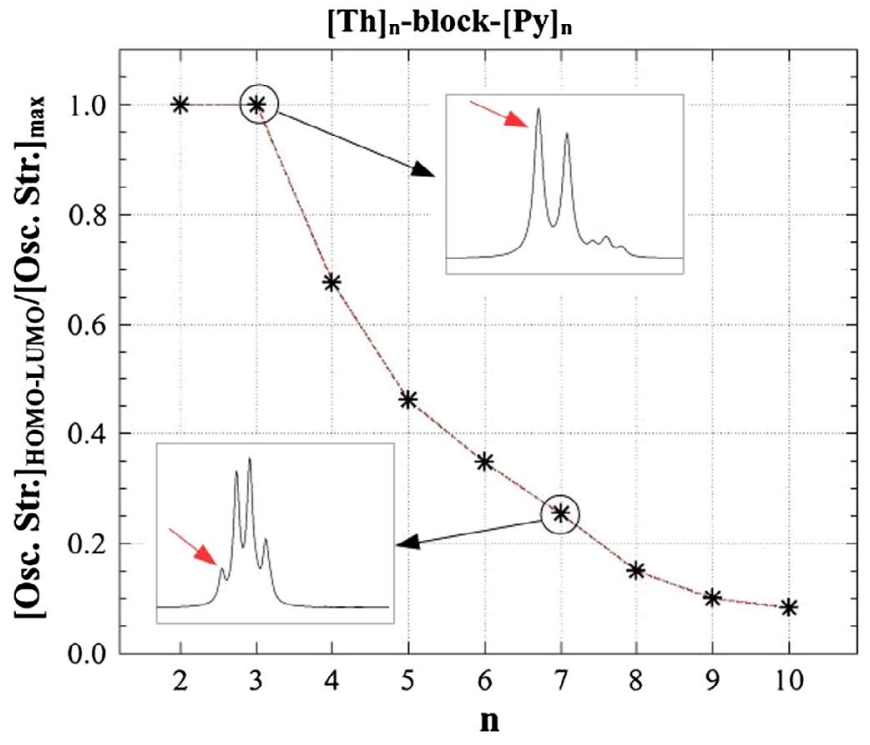

Figure 6. (Colour online) Ratio between the oscillator strengths of the HOMO $\rightarrow$ LUMO and most intense transitions. The insets depict the optical absorption spectrum of selected cases; the red arrows indicate the HOMO $\rightarrow$ LUMO transition.

intense peaks (with a normalised oscillator strength greater than $0.2 \mathrm{au}$ ); the first absorption peak is assigned as a HOMO $\rightarrow$ LUMO transition and the others are combinations of transitions between orbitals below the HOMO and above the LUMO. This suggests that this kind of molecule may provide a wider optical absorption when considering the possible transitions from all conformations accessed when the system changes from the ground to the excited state, with the possible consequence of a higher current in the device $[3,5]$. On the other hand, the $[\mathrm{Th}]_{5}$ and $[\mathrm{Py}]_{5}$ oligomers have both only one intense peak of optical absorption in accordance to what is experimentally observed, that is associated with a $\mathrm{HOMO} \rightarrow$ LUMO transition [45].
Figure 5 shows the orbitals involved in the first four most intense allowed optical transitions for $[\mathrm{Th}]_{5}$-block- $[\mathrm{Py}]_{5}$, and, as can be seen, there is no strong orbital localizations. This is important, because it is known that localised orbitals may act as exciton traps, leaving them with a smaller diffusion length. Looking at the first transition (Figure 5(a), HOMO $\rightarrow$ LUMO), an overlap of only a portion of the involved orbitals was observed. However, the situation is improved for the other transitions in which the orbitals above or below the HOMO and LUMO are involved; they are more delocalized than the frontier ones (see the orbitals HOMO-1, HOMO-2 and LUMO + 2 in Figure 5) so as to allow excitons with larger diffusion lengths $[3,5,45,46]$.

Enlargement of the optical spectrum in diblock materials has been observed previously by Szarko et al. [46], with a theoretical and experimental study of a type of diblock oligomer, different from the DCO that we propose in this work. The authors studied oligomers of $3 \mathrm{HT}$ of the same size connected by a thienothiophene (TT) $\pi$ bridge, resulting in diblock oligomers of different sizes. Analysing the experimental and theoretical optical absorption spectrum of these diblock oligomers, the authors observed a broadening compared to the polymer of $3 \mathrm{HT}$ (poly(3-hexylthiophene), $\mathrm{P} 3 \mathrm{HT}$ ); two intense peaks of optical absorption were present and the first associated to a HOMO $\rightarrow$ LUMO transition. With these results, the authors suggested that these types of materials would have some advantage over the homopolymers, once the P3HT always displays only one intense peak of opti$\mathrm{cal}$ absorption [46]. When increasing the size of $3 \mathrm{HT}$ oligomers in the diblock, the optical spectrum becomes broader, and for oligomers with four monomeric units on each side this effect disappears, and the first most intense peak of optical absorption (the HOMO $\rightarrow$ LUMO transition) starts to lose intensity. Finally, the authors stressed that solar cell efficiency could be improved using the synthesised diblock oligomers, since there would be an increased absorption of the solar spectrum band that would allow more electrons to be excited and possibly contribute to a 
[Th] $]_{3}$-block- $[\mathrm{Py}]_{3}$

LUMO
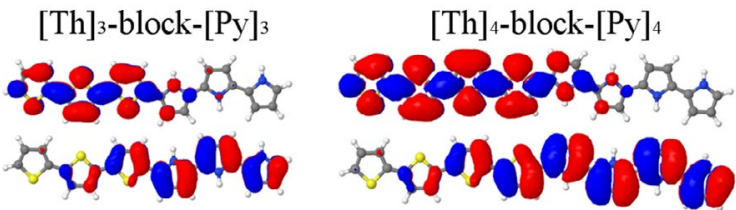

HOMO

[Th] 7-block-[Py]7

LUMO

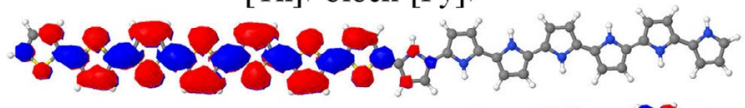

HOMо

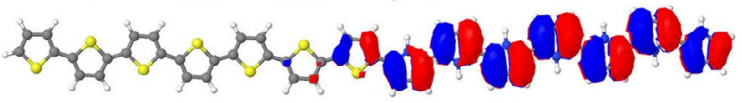

[Th] $\left.]_{10-b l o c k-[P y}\right]_{10}$

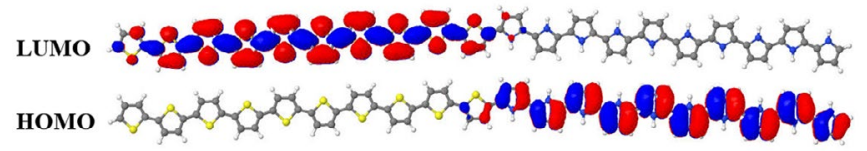

Figure 7. (Colour online) HOMO and LUMO frontier orbitals (Kohn-Sham representation, isovalue surface of $0.01 \mathrm{au}$ ) of $[\mathrm{Th}]_{3}-$ block-[Py $]_{3^{\prime}}[\mathrm{Th}]_{4}$-block-[Py $]_{4^{\prime}}$ $[\mathrm{Th}]_{7}$-block-[Py $]_{7}$ and $[\mathrm{Th}]_{10}$-block-[Py $]_{10}$.

higher current in the device. In our opinion, these experimental results corroborate our predictions for the broadening of the optical absorption band of the DCOs compared to the homooligomers. In addition, for DCOs the results are even more optimised, since more peaks of optical absorption are observed plus the possibility of adjustment of the frontier electronic levels and modulation of the value of $\Delta E_{\mathrm{HL}}$.

When the size of the DCOs is increased, it is possible to observe that, from the point of view of optical absorption, (i) the first peak associated to the HOMO $\rightarrow$ LUMO transition decreases in intensity, and (ii) the optical spectrum becomes simply the sum of the spectra of the two homo-oligomers. Figure 6 shows the oscillator strength intensity evolution of the HOMO $\rightarrow$ LUMO transition for $\mathrm{n}$ ranging from 2 to 10 , relative to the most intense peak of the optical absorption. In the cases that $n=2$ and $n=3$, the most intense transition is the HOMO $\rightarrow$ LUMO itself, and for $n>=4$ it is some other transition. Note that for $n=7$ the $\mathrm{HOMO} \rightarrow$ LUMO transition presents an intensity of only $\sim 25 \%$ compared to the most intense peak. For $n>=7$ the absorption spectrum starts to become similar to the sum of those relating to the homo-oligomers, with the intensity of the first peaks decreasing and tending to disappear (see also Figures S1(f) to S1(i) of SOM). That is, for $n>=7$, the electronic structure of the DCO is no longer a mix of the properties of the diblock constituents: now they are just an overlap of the constituents' properties. This is explained by the decreased overlap of the HOMO and LUMO molecular orbitals for $n>=4$, as shown in Figure 7. This set of results makes clear that there is a size dependency of the electronic and optical properties of DCOs.

We studied other types of DCOs with different blocks built up from $3 \mathrm{HOT}+\mathrm{CNPh}-\mathrm{PV}, 3 \mathrm{HT}-\mathrm{CN}+3 \mathrm{HT}-\mathrm{F}, 3 \mathrm{HT}-\mathrm{CN}+3 \mathrm{HT}$, $3 \mathrm{HT}-\mathrm{OH}+3 \mathrm{HT}-\mathrm{CN}$ and $3 \mathrm{HT}-\mathrm{CN}+3 \mathrm{HT}-\mathrm{NH}_{2}$, following the same procedure used for the DCOs of Th and Py. The results obtained for these other set of DCOs are very similar as for [Th $]_{n}$ block- $[\mathrm{Py}]_{\mathrm{n}}$ and are shown in the SOM.

The set of DCOs from 3HOT + CNPh-PV, 3HT-OH + 3HT$\mathrm{CN}$ and $3 \mathrm{HT}-\mathrm{CN}+3 \mathrm{HT}-\mathrm{NH}_{2}$ exhibited the broader optical absorption spectra of the remaining cases. In these cases, the frontier electronic levels of the constituent oligomers are quite dissimilar. It was observed that the greater the energy differences in $\left|E_{\mathrm{HOMO}, \mathrm{A}}-E_{\mathrm{HOMO}, \mathrm{B}}\right|$ and $\left|E_{\mathrm{LUMO}, \mathrm{A}}-E_{\mathrm{LUMO}, \mathrm{B}}\right|$, the greater the extension of the absorption band. However, due to the large differences between the $E_{\mathrm{HOMO}}$ and $E_{\mathrm{LUMO}}$ of the constituent oligomers, it was also observed that there is a smaller range for $\mathrm{n}$ in which the DCOs are interesting. In these cases, the optimal value for $\mathrm{n}$ is four, however, DCOs with $\mathrm{n}$ equal to three or five can still be interesting. Furthermore, the DCOs with four monomeric units of each homo-oligomer have a $\Delta E_{\mathrm{HL}}$ close to that suggested as ideal $(1.4 \mathrm{eV})[3,13]$.

\section{Conclusions}

This work presents a theoretical study in which we propose the use of diblock co-oligomers (DCO) as small donor molecules for the active layers of OSCs for which it is possible to design the energies of the frontier electronic levels. We verified that it is possible to predict the HOMO and LUMO energies of these types of molecules, knowing beforehand the same values for each of the oligomers employed. Choosing the appropriate oligomers, it is possible to obtain new small molecules with low $\Delta E_{\mathrm{HL}}$ and close to what is considered ideal for applications in solar cells.

The DCOs have a wider optical absorption spectrum when compared to the constituent oligomers and the homopolymers composed of the same monomers. The expected result is a higher conversion efficiency (PCE) as a function of photon harvesting over a wider range of the solar spectrum, with the possibility to produce a higher electrical current in the device. The greatest enlargement of the optical absorption band is obtained for the largest difference between the energies of the frontier electronic levels of the constituent oligomers. There is, however, a size effect; the advantages of the DCOs are observed for a narrow range of the number of monomers.

Overall, the DCOs bring together a number of advantages over the materials commonly used as electron donor elements in active layers of OSCs: the possibility of modelling the HOMO and LUMO energies, the energy difference between the HOMO and LUMO and the enlargement of the optical absorption spectrum. As the field of small molecules-based OSCs is very active, with recent results for PCE around 10\% [6,7], we believe that the DCOs is a class of promising materials.

\section{Acknowledgments}

We would like to thank the Brazilian agency FAPESP (proc. 2012/21983-0 and 2014/20410-1) for financial support. This research was also supported by resources supplied by the Center for Scientific Computing (NCC/ GridUNESP) of the São Paulo State University (UNESP).

\section{Funding}

This work was supported by the FAPESP [proc. 2012/21983-0 and 2014/20410-1].

\section{References}

[1] Li Y. Molecular design of photovoltaic materials for polymer solar cells: toward suitable electronic energy levels and broad absorption. Acc Chem Res. 2012;45(5):723-733. 
[2] Abdulrazzaq OA, Saini V, Bourdo S, et al. Organic solar cells: a review of materials, limitations, and possibilities for improvement. Part Sci Technol. 2013;31(5):427-442.

[3] Dou L, Liu Y, Hong Z, et al. Low-bandgap near-IR conjugated polymers/molecules for organic electronics. Chem Rev. 2015;115(23):12633-12665.

[4] Li G, Zhu R, Yang Y. Polymer solar cells. Nat Photonics. 2012;6:153161

[5] Mishra A, Bäuerle P. Small molecule organic semiconductors on the move: promises for future solar energy technology. Angew Chem Int Ed. 2012;51(9):2020-2067.

[6] Zhang Q, Kan B, Liu F, et al. Small-molecule solar cells with efficiency over 9\%. Nat Photonics. 2014;9:35-41.

[7] Kan B, Li M, Zhang Q, et al. A series of simple oligomer-like small molecules based on oligothiophenes for solution-processed solar cells with high efficiency. J Am Chem Soc. 2015;137(11):3886-3893.

[8] Guo X, Xiao L, Tang W, et al. Synthesis and characterization of a new solution-processable star-shaped small molecule based on 5,6-bis(noctyloxy)-2,1,3-benzoselenadiazole for organic solar cells. J Mater Sci. 2013;48(17):5833-5839.

[9] Yang M, Chen X, Zou Y, et al. A solution-processable D-A-D small molecule based on isoindigo for organic solar cells. J Mater Sci. 2013;48(3):1014-1020.

[10] Zhang L, Shen W, He R, et al. Density functional study on the effect of aromatic rings flanked by bithiophene of novel electron donors in small-molecule organic solar cells. Mater Chem Phys. 2016;175:1321.

[11] Li Y, Guo Q, Li Z, et al. Solution processable D-A small molecules for bulk-heterojunction solar cells. Energy Environ Sci. 2010;3:14271436.

[12] Liu Y, Chen C-C, Hong Z, et al. Solution-processed small-molecule solar cells: breaking the $10 \%$ power conversion efficiency. Sci Rep. 2013;3:3356.

[13] Scharber MC, Sariciftci NS. Efficiency of bulk-heterojunction organic solar cells. Prog Polym Sci. 2013;38(12):1929-1940.

[14] Anslyn EV, Dougherty DA. Modern physical organic chemistry. Sausalito (CA): University Science Books; 2006.

[15] Tanaka K, Shichiri T, Yamabe T. Electronic properties of mislinked polypyrrole and polythiophene. Synth Met. 1986;14(4):271-277.

[16] Oliveira EF, Lavarda FC. Copolymers with similar comonomers: tuning frontier orbital energies for application in organic solar cells. Polym Eng Sci. 2016;56(4):479-487.

[17] Oliveira EF, Lavarda FC. Molecular design of new P3HT derivatives: adjusting electronic energy levels for blends with PCBM. Mater Chem Phys. 2014;148(3):923-932.

[18] Oliveira EF, Camilo-Jr A, Silva-Filho LC, et al. Effect of chemical modifications on the electronic structure of poly(3-hexylthiophene). J Polym Sci, Part B: Polym Phys. 2013;51(10):842-846.

[19] Oliveira EF, Lavarda FC. Reorganization energy for hole and electron transfer of poly(3-hexylthiophene) derivatives. Polymer. 2016;99:105-111.

[20] de Oliveira EF, Lavarda FC. Structure of P3HT in the solid state. J Polym Sci, Part B: Polym Phys 2013;51(18):1350-1354.

[21] Batagin-Neto A, Oliveira EF, Graeff CFO, et al. Modelling polymers with side chains: MEH-PPV and P3HT. Mol Simul. 2013;39(4):309321.

[22] Perepichka IF, Perepichka DF. Handbook of thiophene-based materials. Chichester: Wiley; 2009.

[23] Skotheim TA, Elsenbaumer RL, Reynolds JR, editors. Handbook of conducting polymers. New York (NY): Marcel Dekker; 1998.

[24] Stewart JJP. Optimization of parameters for semiempirical methods $\mathrm{V}$ : modification of NDDO approximations and application to 70 elements. J Mol Model. 2007;13(12):1173-1213.
[25] MOPAC2012 [internet]. Colorado Springs (CO): Stewart Computational Chemistry; 2012. Available from: HTTP://Open MOPAC.net

[26] Jensen J. Introduction to computational chemistry. Hoboken (NJ): John Wiley \& Sons; 2007.

[27] Becke AD. Density-functional thermochemistry. III. The role of exact exchange. J Chem Phys. 1993;98:5648-5652.

[28] Becke AD. A new mixing of Hartree-Fock and local densityfunctional theories. J Chem Phys. 1993;98:1372-1377.

[29] Ditchfield R. Self-consistent molecular-orbital methods. IX. An extended Gaussian-type basis for molecular-orbital studies of organic molecules. J Chem Phys. 1971;54(2):724-728.

[30] Frisch MJ, Trucks GW, Schlegel HB, et al. Gaussian 09. Revision A.01. Gaussian, Inc.; 2009.

[31] Jin R, Wang K. Rational design of diketopyrrolopyrrole-based small molecules as donating materials for organic solar cells. Int J Mol Sci. 2015;16(9):20326-20343.

[32] Bhatta RS, Tsige M. Structural dependence of electronic properties in A-A-D-A-A-type organic solar cell material. Int J Photoenergy. 2015:7p. DOI:https://doi.org/10.1155/2015/708048

[33] Scheuble M, Goll M, Ludwigs S. Branched terthiophenes in organic electronics: from small molecules to polymers. Macromol Rapid Commun. 2015;36(2):115-137.

[34] Tirado-Rives J, Jorgensen WL. Performance of B3LYP density functional methods for a large set of organic molecules. J Chem Theory Comput. 2008;4(2):297-306.

[35] McCormick TM, Bridges CR, Carrera EI, et al. Conjugated polymers: evaluating DFT methods for more accurate orbital energy modeling. Macromolecules. 2013;46(10):3879-3886.

[36] Roldao JC, Oliveira EF, Lavarda FC. Electronic structure of polythieno[3,4-b]-thiophene-co- benzodithiophene (PTB7) derivatives for organic solar cell applications. Org Electron. 2016;33:246-252.

[37] Oliveira EF, Lavarda FC. Effect of the length of alkyl side chains in the electronic structure of conjugated polymers. Mat. Res. 2014;17(6):1369-1374

[38] Yang S, Olishevski P, Kertesz M. Bandgap calculations for conjugated polymers. Synth Met. 2004;141(1-2):171-177.

[39] Gierschner J, Cornil J, Egelhaaf H-J. Optical bandgaps of $\pi$-conjugated organic materials at the polymer limit: experiment and theory. Adv Mater. 2007;19(2):173-191.

[40] Yang Y-L, Lee Y-H, Lee Y-P, et al. Synthesis and characterization of poly(3-hexylthiophene)-poly(3-hexyloxythiophene) random copolymers with tunable band gap via Grignard metathesis polymerization. Polym Int. 2014;63(12):2068-2075.

[41] Tummala NR, Risko C, Bruner C, et al. Entanglements in P3HT and their influence on thin-film mechanical properties: Insights from molecular dynamics simulations. J Polym Sci, Part B: Polym Phys. 2015;53(13):934-942.

[42] Alexiadis O, Mavrantzas VG. All-atom molecular dynamics simulation of temperature effects on the structural, thermodynamic, and packing properties of the pure amorphous and pure crystalline phases of regioregular P3HT. Macromolecules. 2013;46(6):24502467.

[43] Marques MAL, Ullrich CA, Nogueira F, et al. Time-dependent density functional theory. Heidelberg (BW): Springer; 2006.

[44] Jmol: an open-source Java viewer for chemical structures in 3D. Available from: http://www.jmol.org/

[45] Milián-Medina B, Gierschner J. $\pi$-conjugation. Wiley Interdiscip Rev Comput Mol Sci. 2012;2(4):513-524.

[46] Szarko JM, Rolczynski BS, Guo J, et al. Electronic processes in conjugated diblock oligomers mimicking low band-gap polymers: Experimental and theoretical spectral analysis. J Phys Chem B. 2010;114(45):14505-14513. 